\title{
Strates
}

STRATES Matériaux pour la recherche en sciences sociales

14 | 2008

Espaces du quotidien

\section{Les territoires invisibles et vitaux de la lecture}

\section{Michèle Petit}

\section{(2) OpenEdition}

\section{Journals}

Édition électronique

URL : http://journals.openedition.org/strates/6734

DOI : $10.4000 /$ strates. 6734

ISSN : 1777-5442

Éditeur

Laboratoire Ladyss

Édition imprimée

Date de publication : 1 janvier 2008

Pagination : 213-224

ISBN : 0768-8067

ISSN : 0768-8067

Référence électronique

Michèle Petit, «Les territoires invisibles et vitaux de la lecture », Strates [En ligne], 14 | 2008, mis en ligne le 05 mars 2013, consulté le 08 septembre 2020. URL : http://journals.openedition.org/strates/ 6734 ; DOI : https://doi.org/10.4000/strates.6734

Ce document a été généré automatiquement le 8 septembre 2020

Tous droits réservés 


\title{
Les territoires invisibles et vitaux de la lecture
}

\author{
Michèle Petit
}

Dans l'Invention du quotidien, consacrée aux créativités, fugues et ruses des « hommes ordinaires ", Michel de Certeau s'attachait à déceler l'irréductible part de liberté existant dans les univers les plus quadrillés et disciplinés. Le quotidien était construction, interactions, opérations poétiques; il ne pouvait être confondu avec l'aliénation, les assignations, la passivité. La recherche dont $\mathrm{M}$. de Certeau rendait compte était plus particulièrement dédiée aux pratiques de l'espace, aux manières de fréquenter un lieu, mais pour les décrire, un point de départ s'était imposé à lui : l'analyse de la lecture, activité méconnue, production silencieuse. Tout comme les lecteurs s'introduisent dans les textes lus, les détournent, y braconnent du sens, les rendant habitables "à la manière d'un appartement loué ", les citadins arpentent le texte de la ville qu'ils n'ont pas écrit, de façon récurrente, mais jamais identique. Si contraignante soit-elle, une nouvelle forme d'urbanisation finit, tel un livre, par être reconstruite et réinventée par des cheminements, des récits, un travail de mémoire (Certeau, 1980).

2 Le livre pensé comme un espace habitable, la ville conçue comme un texte, tout au long de cet ouvrage inclassable, M. de Certeau jouait de cette mise en résonance. Au-delà du bonheur heuristique des métaphores, l'étude des « arts de faire » mis en œuvre dans la lecture conduit à questionner les acceptions courantes du quotidien et de ses territoires, et à s'étonner de leur réduction fréquente au banal ou au proche. C'est du moins ce que suggère l'analyse des appropriations opérées par celles et ceux qui ont rencontré des textes, ou des fragments de textes, alors qu'ils vivaient au plus loin des livres: dans des espaces en crise, qu'ils soient le cadre de guerres ou de violences répétées, de déplacements forcés de populations ou de déqualifications économiques rapides ${ }^{1}$; dans des quartiers stigmatisés en périphérie urbaine, ou des régions rurales éloignées des bibliothèques et des librairies ${ }^{2}$. De leurs expériences, des traces peuvent être retrouvées dans les récits, oraux ou écrits, qu'ils font de ces rencontres, quelquefois dans un simple souvenir ou une anecdote : toutes narrations qui, par les 
mises en rapport qu'elles établissent, permettent de cerner le sens que prend cette pratique.

\section{Un espace en rupture avec le cadre et les activités ordinaires}

3 Anibal Luis Meléndez vit dans l'État de Oaxaca, au Mexique ; il est enseignant. Dans un texte où il rassemble ses souvenirs de lecteur, il raconte un jour, à l'époque où il allait au jardin d'enfants, où quelqu'un offrit à son père un livre illustré «bleu comme le ciel »:

[...] je me souviens que mon père le tenait dans ses mains, le regardait fixement, allongé dans le hamac qu'il accrochait aux murs de la chambre où nous dormions. J'ai réussi à voir quelques dessins et quantité de choses curieuses, plus tard j'ai su que c'étaient des lettres. Ce livre suscitait ma curiosité car l'attitude de mon père changeait quand il s'y confrontait, il ne se comportait pas comme tous les jours ${ }^{3}$.

4 En Amérique latine comme en Espagne ou en France, dans des autobiographies de lecteurs ${ }^{4}$, la mention d'une telle scène revient: ce qui a alerté un enfant, suscité sa curiosité pour l'écrit, c'est ce comportement inhabituel de la mère, du père, de la grand-mère ${ }^{5}$ ou d'un proche. Quand bien même se répétait-il jour après jour, ou plutôt soir après soir, il différait de façon inexplicable de celui que ces adultes affichaient le reste du temps, introduisait une rupture dans les usages ordinaires.

De fait, dans des espaces où les interdits culturels se sont ajoutés aux obstacles sociaux et géographiques pour entraver l'accès aux livres, ceux-ci sont perçus comme des objets étranges, insolites, voire incongrus, qui fascinent les uns, inquiètent les autres, et finissent par être circonscrits en des lieux contrôlés - placard sous clé, caisse, cagibi. Des objets dont les pouvoirs de distraire, au sens fort du terme, celles et ceux qui s'y abandonnent, intriguent les enfants, qui tentent de s'en saisir pour découvrir leurs secrets et s'emparer de leur puissance.

6 Bien entendu, selon les contextes culturels, l'ampleur de la pauvreté, les niveaux de scolarisation, les spécificités de l'histoire locale, les héritages familiaux, les singularités individuelles, etc., l'intensité des pratiques de lecture diffère, tout comme les représentations de l'écrit, du livre. Là où la plupart des habitants n'ont pas pu poursuivre un cursus scolaire au-delà de quelques années, où le temps est largement dédié à la survie, où le groupe prend le pas sur celles et ceux qui le constituent à chaque moment ou presque, la lecture est quasi inexistante. Mais plus largement, en milieu populaire rural ou urbain, de part et d'autre de l'Atlantique, elle trouve difficilement sa place parmi les activités effectuées au quotidien. En effet, son « utilité » n'est pas bien établie - sauf quand on lit à des fins scolaires, professionnelles ou pratiques. À l'époque contemporaine, cette pratique suppose habituellement de se tenir à l'écart des autres, et un tel souci de soi, un tel détachement, peuvent être jugés grossiers là où l'on privilégie les activités partagées, sinon dans les faits, du moins dans les principes. Se confronter directement aux livres, c'est encore manquer à sa condition, singer les "lettrés », voire les anciens colonisateurs. C'est donc souvent hors du cadre de vie habituel que des gens en sont venus à lire, à l'occasion d'un séjour en pension ou à l'hôpital, par exemple ; ou bien c'est un passeur ayant vécu dans d'autres milieux qui a introduit des livres et rendu leur appropriation désirable (Ladefroux et al., 1993 ; Petit et al., 1997). 
7 Dans ces contextes, lire n'est jamais vraiment légitime. C'est un geste de l'ombre qui s'effectue discrètement, loin du regard des autres. S'il est le fait d'une femme, il a partie liée avec une quasi-clandestinité (Sayad, 1995) et des «temps dérobés " (Thiesse, 1995) repris au temps social; ou bien il s'accomplit dans le cadre de sociabilités censées bénéficier, indirectement, à la réussite scolaire des enfants (Petit, 2007). S'il est le fait d'un garçon, il suppose souvent de se cacher pour échapper aux sarcasmes des pairs et préserver l'espace du désir à l'heure où la lecture devient corvée, exercice convenu pour satisfaire les adultes.

8 Car la dimension transgressive, hors du commun, est aussi ce qui fait le charme de la lecture, et sa valeur. Pour ceux qui vivent dans des campagnes reculées, des quartiers stigmatisés, ou plus encore des espaces en crise, et qui ont pu s'approprier des livres ou des fragments de textes, cette expérience est vécue comme un geste de sortie, une rupture, un saut hors du cadre habituel. Par le biais d'une légende, d'un poème, d'une fiction ou d'un essai, ils disent avoir pu quitter le face à face avec trop de réel, le ressassement; passer ailleurs. Nombreuses sont les métaphores employées qui évoquent un envol, un mouvement ascensionnel euphorique, particulièrement dans des contextes violents où une part d'eux échappe alors à la loi du lieu ou aux conflits récurrents. Aussi divers professionnels - bibliothécaires, psychologues, enseignants... recourent-ils délibérément à la lecture, fréquemment oralisée, pour aider des populations vivant dans de tels contextes à se dégager et acquérir une marge de manœuvre (Petit, 2008).

9 Toutefois, dans des milieux plus nantis en capital culturel légitime, où l'appropriation du livre semble aisée, banalisée, bien des pratiques indiquent que celles et ceux qui s'adonnent à cette activité tentent de réintroduire de l'ombre, du secret. Ainsi, dans des familles où l'on n'a jamais interdit de lire et où des livres sont proposés aux enfants, ceux-ci lisent quelquefois sous les draps, lampe de poche à la main, pour préserver à la lecture sa part clandestine, transgressive. Et là encore, ce qui est trouvé, avant tout, c'est un espace, en rupture avec le cadre et les activités ordinaires. Expérience particulièrement recherchée pour accompagner le passage de la veille au sommeil ${ }^{6}$, mais aussi celui d'un lieu à un autre - il n'est que de penser à tous ceux qui lisent en empruntant un moyen de transport.

10 "Expérience initiale, voire initiatique », qui valait, selon M. de Certeau, pour les lettrés comme pour les lecteurs de milieux populaires: «lire, c'est être ailleurs, là où ils ne sont pas, dans un autre monde; c'est constituer une scène secrète, lieu où l'on entre et d'où l'on sort à volonté ; c'est créer des coins d'ombre et de nuit dans une existence soumise à la transparence technocratique... » (1980, p. 291).

\section{"Un espace dans lequel on peut évoluer »}

11 On lirait donc sur les bords, les rivages de la vie - sauf quand la lecture est purement utilitaire. Le quotidien visible aurait là son envers, ses plages dérobées où opérer des passages, prendre de la distance de temps à autre, respirer. Toutefois, il ne s'agit pas d'une fuite, comme il est dit souvent de façon un peu dépréciative, comme s'il était plus honorable d'être tout à sa douleur ou à son ennui. Si l'on analyse les récits des lecteurs, l'espace en rupture ouvert par cette pratique apparaît doté de plusieurs 
caractéristiques, indiquant qu'il est propice au déploiement de soi et à l'ouverture à l'autre, bien plus qu'au repli :

- il est habituellement associé à la découverte d'un univers radicalement autre, d'un lointain, légendaire ou exotique - la jungle, lîle des mers du Sud, une autre galaxie, pour qui vit dans des pays du Nord ; la neige, la clairière d'un bois frais d'Europe, les châteaux forts, quand on est en Amérique tropicale. Ce lointain des lectures vient modifier la perception des lieux familiers, les ouvre à de l'ailleurs. Comme pour cette femme, Martha, habitant au nord de l'Argentine, qui, regardant les sommets découpés près de la Puna Jujeña, y voit « les forteresses médiévales européennes » dont elle s'est « saisie dans les livres »;

- il est décrit comme un espace habitable : à partir de ces pays lointains, c'est une « chambre à soi » qui s'esquisse, y compris dans des contextes où aucune possibilité de disposer d'un espace personnel ne semblait exister - en prison, par exemple. Fréquemment, les métaphores utilisées renvoient à un lieu d'accueil, à l'hospitalité, à la maison ou à la terre retrouvée pour qui a vécu un exil (« la lecture, c'est mon pays »). Les livres sont autant de demeures empruntées, le moyen de recréer par soi-même le sol perdu - du reste, c'est peutêtre avant tout à la part exilée de chacun que les livres, et plus encore la littérature, s'adressent (Petit, 2008).

12 Aussi ces objets sont-ils parfois utilisés avec des populations déplacées, frappées par des catastrophes naturelles ou des conflits armés, dont le cadre de vie a été perdu, détruit ou profondément altéré, comme c'est le cas en Colombie, en périphérie de Medellín, où des bibliothécaires ont développé un programme intitulé « Le refuge des contes » quand une partie de la population a été chassée par les combats entre groupes armés. Consuelo Marín (2004b) évoque ainsi un matin où elle lisait à voix haute dans le lycée où la population avait été regroupée et où elle entendit des tirs qui se rapprochaient au point qu'elle voulut s'interrompre, mais les auditeurs exigèrent d'entendre la fin: " ces enfants qui passaient leurs nuits en pleurant dans les couloirs du lycée, en redoutant le noir, ne voulaient pas perdre la fin du conte, telle une seconde peau, peau de l'âme que l'on ne peut pas enlever ${ }^{7}$ ";

- pour qui vit dans un environnement chaotique, il permet de retrouver un sentiment de continuité. Y concourent la régularité, la permanence du recours possible, tout comme la structure de l'objet - le codex, fait de feuilles reliées - et des narrations qu'il abrite - dotées d'un début, d'un développement, d'une fin. Y contribue encore le fait que la lecture déclenche une activité de symbolisation, de pensée, de narration de sa propre histoire entre les lignes lues, un tissage de liens entre des épisodes vécus de façon fragmentée (Ray Bradbury disait des livres qu'ils « cousent les pièces et les morceaux de l'univers pour nous en faire un vêtement ${ }^{8} »$ ). Toutes choses qu'observent les médiateurs développant des ateliers fondés sur cette pratique dans des régions frappées par des conflits armés (Petit, 2007).

- « seconde peau » ou " vêtement », c'est encore à une délimitation de soi, à un tracé de ses contours que l'espace ouvert par la lecture semble propice. Les lecteurs s'y sentent reliés aux autres - aux personnages, à l'auteur, à ceux qui ont lu le livre ou le liront - dont ils découvrent qu'ils partagent les mêmes émotions ou les mêmes désarrois, mais ils s'y perçoivent comme séparés, différents de ce qui les entoure, capables d'une pensée indépendante;

- cet espace est réglé par un tempo particulier, en rupture, là encore, avec les autres activités, fait de lenteur propice à la rêverie, mais aussi, quelquefois, d'un rythme plus proche du sensoriel ; et il est le lieu d'une autre langue que celle utilisée pour la désignation immédiate 
et utilitaire des choses : la langue du récit, dont on redécouvre depuis une vingtaine d'années combien elle nous constitue (Bruner, 2002);

- par ces décalages, ces décrochements, l'espace ouvert par la lecture introduit du jeu et une créativité, d'autant qu'il est aussi un lieu où se ressourcer, où alimenter sa vitalité. Au-delà du caractère enveloppant, protecteur, habitable, de la lecture, une transformation des émotions et des sentiments, une élaboration symbolisée de l'expérience vécue, une projection dans un futur et une entrée en relation avec d'autres sont, dans certaines conditions, rendues possibles. Pour reprendre l'expression utilisée par un garçon dans un quartier dit « sensible », c'est « un espace dans lequel on peut évoluer », un lieu de redéploiement des possibles. Le monde n'en est évidemment pas pour autant réparé de ses drames, de ses conflits ou de ses inégalités, mais une marge de manœuvre est ouverte.

Sous l'angle du quotidien, la lecture s'avère donc paradoxale. C'est une activité s'effectuant fréquemment dans des temps dérobés, mais qui revient comme un rituel, de façon quasi vitale. En rupture avec les autres gestes qui se répètent jour après jour (et avec ceux que l'on côtoie), elle est le moyen de retrouver une continuité quand celle-ci fait défaut, d'éprouver simultanément un lien aux autres et le sentiment de son individualité. Elle permet de s'arracher à l'enfermement, aux assignations, au(x) proche(s); pourtant c'est sans doute l'une des pratiques (un peu comme planter des fleurs dans un potager ou faire une excursion ?) qui font que du quotidien est possible. Et objet de créativité.

\section{Une aire intermédiaire d'expérience, entre intérieur et extérieur, proche et lointain}

14 Telle est l'expérience évoquée, sous des formes multiples, par nombre de celles et ceux vivant dans des espaces en crise qui ont pu s'approprier des textes ou des bribes de textes. Et au-delà, par bien des lecteurs de différents milieux culturels ou sociaux. Ils retrouvent là, en fait, une expérience originaire décisive, décrite par Donald W. Winnicott à l'aide du concept d'" espace transitionnel ", qui éclaire le statut paradoxal de la lecture et permet de mieux situer les enjeux anthropologiques et psychiques de ces pratiques à la lisière du quotidien.

Pour D. W. Winnicott (1975), l'espace transitionnel désigne une aire de jeu qui s'inaugure entre le jeune enfant et sa mère, si l'enfant se sent en confiance. Il se constitue dans les moments où celui-ci s'approprie quelque chose que sa mère lui propose pour se différencier d'elle. Par exemple, il reprend la chanson qu'elle lui chante ou la comptine qu'elle lui dit, et grâce aux syllabes ou à la mélodie incorporées qui le protègent, il se lance, s'éloigne un peu: espace psychique ${ }^{9}$ plus que matériel, l'aire transitionnelle se construit aussi avec le corps qui se déplace, par une exploration d'un monde physique progressivement élargi. Par ces allées et venues, l'enfant esquisse ses propres contours. La chanson ou la comptine rétablissent une continuité et permettent que l'angoisse de séparation soit surmontée. Progressivement, il élabore sa capacité d'être seul, construit l'espace du secret, d'une pensée indépendante, d'une aptitude à entrer en relation avec d'autres, au-delà de l'union première. Au lieu même où la séparation a lieu, s'ouvre le champ de la symbolisation, du jeu, puis de l'art et de la culture : si l'on suit D. W. Winnicott, les expériences culturelles sont une extension de ces premières ébauches de vie créatrice, d'émancipation. 

les passages. Dans les situations de crise, scandées de ruptures, de séparations ou d'exils, la recréation d'une telle aire serait fondamentale pour être à même d'établir des liens avec le monde intérieur comme avec le monde extérieur, retrouver sa faculté de symboliser, de penser, de créer. Dans la lecture, quand elle n'est pas régie par l'obligation, c'est cet espace esthétique, calme, protecteur où se ressourcer et «travailler » la séparation qui serait reconstitué. Mais bien entendu, les expériences culturelles débordent le domaine de la littérature ou de l'art consacré. Pour D. W. Winnicott (comme pour M. de Certeau), « une création c'est un tableau, une maison, un jardin, un vêtement, une coiffure, une symphonie, une sculpture, et même un plat préparé à la maison » (1977, p. 61). Universelle, inhérente au fait de vivre, la créativité est pour lui ce "qui permet à l'individu l'approche de la réalité extérieure »: on ne peut approcher celle-ci qu'en la créant, en l'apprivoisant. pour que les enfants s'y défoulent avant de revenir aux choses sérieuses, D. W. Winnicott pensait que ce que l'on fait de sérieux, avec créativité, trouve son origine dans le jeu, dans la culture, et que c'est dans l'espace transitionnel, potentiel, que s'opère le vrai travail. Mais cette créativité ne surgit pas à partir de rien, elle suppose que des ressources soient mises à la disposition de l'enfant (et de l'enfant qui continue à vivre en chacun, tout au long de la vie) ${ }^{10}$.

Ce que suggère l'expérience des lecteurs, c'est qu'elle suppose également de rencontrer quelque chose qui ouvre sur un ailleurs, sur un lointain temporel (et ce sont par exemple les légendes, orales, écrites, ou inscrites dans des monuments), ou un lointain géographique.

19 La meilleure connaissance des processus de formation de la subjectivité et du développement de la pensée, grâce aux apports de la psychanalyse et de la psychologie cognitive, contribue, là encore, à une explicitation. Dès leur plus jeune âge, les enfants se construisent par un quotidien fait d'une oscillation entre proche et lointain, entre connu et inexploré; il ne se résume pas au familier. Très tôt, ils évaluent les distances et les angles et divisent « tout espace en deux zones distinctes : un monde proche, à la portée de leur bras tendu, et un monde lointain, au-delà »(Stern, 2004, p. 31). À douze mois, ils agissent selon deux tendances essentielles: l'une, qui relève du système de l'attachement et pousse vers le havre familier ; l'autre, du système de l'exploration, de la curiosité que le monde leur inspire. Toutes choses qui se déclinent évidemment selon des modalités propres aux cultures, aux milieux sociaux, à l'histoire familiale, aux singularités des destins individuels.

20 C'est cette oscillation qui est retrouvée dans la lecture - à la condition que l'on s'y adonne sans trop de contraintes. Lire a à voir avec la liberté d'aller et venir, avec la possibilité d'entrer à volonté dans une autre scène et d'en sortir, dont parlait M. de Certeau dans la phrase citée plus haut. Comme pour le héros de À bout d'enfance où Patrick Chamoiseau a transposé sa propre expérience de jeune garçon antillais (2005, p. 32-33):

Chaque image d'un livre était un monde touché par l'infini, chaque image lui ouvrait d'autant mieux l'infini qu'elle n'entretenait aucun rapport avec son entourage [...] Le négrillon s'envolait par ces fenêtres ouvertes, revenait à ses affres, puis s'en allait encore, jusqu'à condamner les gens de ces illustrations à vivre ses propres sentiments... Il les animait de ses envies. Les remplissait de son mal-être [...] ces gens illustrés prenaient en charge sa poisse mentale par le biais de mille 
mésaventures [...] C'est à travers ces personnages disséminés au fond de lui qu'il vit en face (sans encore les connaître) la mort, la douleur, la peur, les tortures, les abandons, les trahisons et autres catastrophes ordinaires. telles allées et venues, celles-ci semblent largement facilitées quand images et mots n'entretiennent aucun rapport avec l'entourage, pour parler comme P. Chamoiseau. Et ce, quel que soit le milieu socioculturel. On s'étonne d'autant que certains médiateurs tentent de cantonner leurs « publics » à des textes supposés leur ressembler.

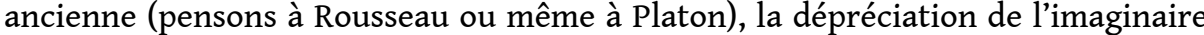
et de la fiction, vue comme des égarements trompeurs hors d'une « réalité » confondue avec l'environnement matériel proche, a pesé de façon récurrente sur le modèle de la "bonne » lecture et de la "bonne » littérature pour enfants. Récemment encore, comme l'écrit Graciela Montes (2001, p. 21), "poussèrent comme champignons les histoires d"enfants comme toi', se déroulant dans des situations quotidiennes, semblables dans tout ce qui était visible à celles que connaissait le lecteur - des histoires en déguisements réalistes [...] Les pédagogues étaient contents, parce que l'histoire informait l'enfant sur ce qui l'entourait, elle l'éduquait' (fin ultime de tout ce qui touche à l'enfant) et ne s'égarait pas dans ces obscurs et imprévisibles couloirs de la fantaisie ». Fantaisie qui a pourtant des enjeux considérables...

\title{
Deux registres de la langue
}

\begin{abstract}
Quand j'étais petite, ma maman me racontait, le soir, la lumière éteinte, l'histoire d' Alice au pays des Merveilles [...] elle tenait un petit commerce dans notre maison et, probablement pour cette raison, les aventures de cette Alice, qu'elle me contait, se déroulaient dans un monde d'arbres en chocolat Jack et de cascades de Fanta Orange et de Coca Cola. Je sais qu'Alice arrivait à ce paradis en passant par un miroir (c'est pourquoi j'aimais l'armoire à pharmacie de la salle de bains) et je sais qu'il y avait le lapin et la Reine de Cœur [...] tous les soirs, un monde parallèle naissait dans la voix de ma mère. Avec son récit, je traversais le miroir et j'entrais rituellement dans la fiction. Comme j'y entrais quand elle me racontait l'histoire du roi David ou de mon arrière-arrière-grand père carabinier dans le sud de l'Italie ; l'histoire de Pierre et le Loup et aussi celle de mon oncle Oreste ; les histoires de mes arrière-grands-parents instituteurs en Patagonie au début du siècle et celle de la pierre qui bouge de Tandil près de laquelle ma grand-mère enseignait (Seoane, 2004).
\end{abstract}

Chaque soir, de façon rituelle, la mère de Silvia, à Buenos Aires, tissait des narrations qui transformaient le quotidien, le mêlaient à une dimension poétique. Elles ouvraient la place de l'Autre, introduisaient la petite fille au temps historique du siècle écoulé comme au temps biblique du roi David, agrandissaient sa vie à un espace qui s'étendait jusqu'à la Patagonie et au sud de l'Italie.

Le quotidien des jeunes enfants est également fait de l'oscillation entre deux registres $d u$ langage, le langage en situation, factuel, utilitaire, qui sert à la désignation, et le langage hors situation, celui du récit, qui relate les événements à distance et introduit un autre rapport au temps. Ou du moins est-ce souhaitable pour bien augurer de leur développement psychique comme de leur devenir scolaire (Cabrejo-Parra, 2002; Bonnafé, 2001). "Il semble que nous ayons dès le début de la vie une sorte de prédisposition au récit ", si l'on suit Jerome Bruner (2002); mais celle-ci doit être soutenue pour se déployer. 
Aujourd'hui, on sait en particulier que dès leur plus jeune âge, les enfants ont besoin de littérature pour grandir, pour commencer à formuler leur propre histoire. Tous les grands spécialistes de la petite enfance ont dit combien étaient précieux les moments où, jour après jour, la mère s'adonne avec son bébé à un usage ludique, gratuit, fictionnel, poétique, de la langue ${ }^{11}$. Pour jouer en lui-même avec les situations et les personnes qui l'entourent et symboliser l'absence, pour se penser, peu à peu, comme un petit sujet distinct de sa mère, le jeune enfant doit avoir accès à « un langage qui ne se réduit pas aux noms des choses, et (à) une relation vraie médiatisée par du semblant et des fictions" (Flahault, Heinich, 2005, p. 10). Ce que disait aussi René Diatkine: les histoires lues à l'enfant avant qu'il ne s'endorme lui permettent de mieux supporter le noir, la séparation d'avec les parents, la peur de les perdre, et celle de mourir ; mais il précisait :

Seule une histoire fictive racontée, un récit dans une langue d'une tout autre structure que le parler relâché de la vie quotidienne semble faire effet contre cette angoisse de la séparation (Hommage à René Diatkine, 1999, p. 8).

Du reste, dans toute culture existent des berceuses, des comptines, et des mythes, des contes, des légendes : de la littérature. Grâce aux premières, la prosodie de la langue se transmet, et par le biais des autres, les enfants pourront construire du sens en se saisissant de métaphores.

Toutefois, dans des situations de crise, les registres de la langue se réduisent, tout comme les temps d'échanges gratuits, l'espace de la rêverie, l'imaginaire. Se sentant menacés par l'extérieur, certains transforment leur famille en forteresse (Sayad, 1995). D'autres ont vécu des catastrophes, la mère n'a pas pu chanter, rire, jouer, parler gratuitement avec ses enfants. Ou bien la dimension de l'utile prime dans tous les temps de la vie, il n'y a pas de rapport ludique avec des objets et le langage. Aussi divers professionnels, là encore, s'emploient-ils à une thérapeutique culturelle des subjectivités et des socialités altérées, telles ces conteuses professionnelles, en Argentine, qui, dans des zones de grande pauvreté, permettent à des femmes de retrouver des récits ou des chants oubliés de leur propre enfance et d'élaborer un lien affectif et linguistique plus riche avec leurs bébés (Schlemenson, 2005).

Au-delà de l'enfance, ce que l'analyse de l'expérience des lecteurs fait apparaître, c'est combien il a été pour eux précieux de doubler leur rapport immédiat à la réalité objective, matérielle, d'une médiation symbolique et métaphorique. De trouver hors d'eux-mêmes des figurations leur permettant de mettre en scène, de façon distanciée, ce qu'ils avaient vécu, relançant leur propre narration intérieure et lui donnant, quelquefois, un fil rouge.

Bien entendu, d'un contexte à l'autre, les formes de la narrativité, les rapports entre oral et écrit, le statut de la parole et du silence diffèrent. Toutefois, quel que soit le milieu culturel et social, la fonction réparatrice des récits fictifs est manifeste (qu'ils relèvent de la littérature orale ou écrite). Didier Anzieu pensait que par le rêve, on recréait, chaque nuit, l'enveloppe psychique vitale que les petits traumatismes de la journée avaient criblée de trous. Les récits de fiction réparent, au quotidien, les déchirures et apprivoisent l'étrange, l'inquiétant. La mise en ordre séquentielle, l'élaboration esthétique dont ces narrations ont fait l'objet, rassurent: on n'est plus dans le désordre, les événements contingents prennent sens dans une histoire mise en perspective. Et c'est un peu comme si par l'ordre secret qui en émane, le chaos du monde, intérieur ou extérieur, pouvait prendre forme. 


\section{(1)} ienne. Zina, une jeune femme écoutée par Abdelmalek Sayad, utilisait presque les mêmes mots : elle lisait " pour exister autrement que de manière végétative ", "pour ne pas se laisser détruire ». Il s'agissait d'une véritable « entreprise de survie » dans un contexte de claustration familiale et de grande solitude (Sayad, 1995). Sous des formes multiples, dans des situations de crise, de tels actes de résistance d'hommes et de femmes se retrouvent. Ceux-ci font flèche de tout bois, de tout texte - ou toute image susceptible d'ouvrir l'horizon, pour résister à l'enfermement, aux assignations à demeure et aux éventuelles tentatives des pouvoirs, politiques, symboliques ou domestiques, d'entraver, resserrer et contrôler leurs mouvements ${ }^{12}$. Ils s'efforcent de sauvegarder une intelligence d'eux-mêmes et du monde et de retrouver un lien avec ce lointain intérieur où l'on s'ouvre aux autres. De préserver envers et contre tout une dignité et une part de liberté, de rêve, d'inattendu.

\section{BIBLIOGRAPHIE}

Anzieu D., 1995, Le Moi-peau, Paris, Dunod. 
Baudelot C., Cartier M., Détrez C., 1999, Et pourtant, ils lisent..., Paris, Seuil.

Bonnafé M., 2001, Les livres, c'est bon pour les bébés, Paris, Calmann-Lévy.

Bruner J., 2002, Pourquoi nous racontons-nous des histoires ? Paris, Retz.

Cabrejo-Parra E., 2002, « Langue, littérature et construction de soi », in Zoughebi H. (coord.), La littérature dès l'alphabet, Paris, Gallimard, p. 69-85.

Certeau M. de, 1980, L'invention du quotidien, 1) Arts de faire, Paris, 10/18.

Chamoiseau P., 2005, À bout d'enfance, Paris, Gallimard-Haute Enfance, 2005.

Détrez C., 2000, « Du côté des lecteurs et des pratiques de lecture », in Mollier (dir.), Où va le livre ? Paris, La Dispute, p. 177-189.

Flahault F.,Heinich N., 2005, « La fiction, dehors, dedans », L’Homme, n 175-176, juill.-déc.

Hommage à René Diatkine, Les Cahiers ACCES, 4, 1999.

Ladefroux R., Petit M. et al., 1993, Lecteurs en campagnes, Paris, BPI/Centre Georges Pompidou, coll. «Études et recherches ».

Marín C., 2004a, « Los programas bibliotecarios para jóvenes en el contexto de la guerra urbana », Nuevas hojas de lectura, 6, p. 35-41.

www.eleducador.com/.../HOME/CHILE/SECCPRINCIPALES/PUBLICACIONES/HOJAS/6/ EXPERIENCIAS

Marín C., 2004b, « Biblioteca pública, bitácora de vida », www.anabad.org/archivo/ docdow.php ? id $=39$

Michel X., 2006, « Écrire les espaces de présence éphémère. Géographie des incursions vers les lieux du patrimoine ", thèse de doctorat sous la direction d'Y. Luginbühl, Université de Paris 1.

Montes G., 2001, El corral de la infancia, Mexico, Fondo de cultura económica.

Petit M., 2002, Éloge de la lecture. La construction de soi, Paris, Belin, coll. « Nouveaux mondes ».

Petit M., 2004a, « Les pays lointains de la lecture », Ethnologie française, XXXIV, nº 4, p. 609-615.

Petit M., 2004b, « Une expérience littéraire partagée au village : “Lire ensemble”, à Ballobar (Espagne) », journées d'études franco-helléniques « Numérisation, lien social, lectures », Université de Crète, Rethymnon (Grèce).

Petit M., 2007, « La littérature comme médiation : quelques expériences dans des régions en guerre », Médiations, médiateurs, médias, actes du colloque du Salon du livre et de la presse Jeunesse, Montreuil, CPLJ.

Petit M., 2008, Un art de lire par temps de crise, Paris, Belin, coll. « Nouveaux mondes ». (À paraître.)

Petit M., Balley C., Ladefroux R., avec la collaboration de Rossignol I., 1997, De la bibliothèque au droit de cité, Paris, BPI/Centre Georges Pompidou, coll. «Études et recherches ».

Sayad A., 1995, « La lecture en situation d'urgence », in Seibel B. (dir.), Lire et faire lire, Paris, Le Monde Éditions, p. 65-99.

Schlemenson S., 2005, El placer de criar, la riqueza de pensar, Buenos Aires, Novedades educativas. Seoane S., 2004, « Tomar la palabra. Apuntes sobre oralidad y lectura », Conférence présentée dans le cadre du Postítulo de Literatura Infantil y Juvenil, Buenos Aires, CePA.

Stern D., 2004, Journal d'un bébé, Paris, Odile Jacob. 
Thiesse A.-M., 1995, « Organisation des loisirs et temps dérobé (1830-1930) », in Corbin A. (dir.), L'avènement des loisirs, 1850-1960, Paris, Aubier, p. 302-322.

Winnicott D. W., 1975, Jeu et réalité, Paris, Gallimard.

Winnicott D. W., 1977, L'arc, 69.

\section{NOTES}

1. Par " espaces en crise ", on désigne des espaces où des changements à caractère brutal même s'ils ont été préparés sur le temps long - ou encore une violence durable et omniprésente, rendent largement inopérants les modes de régulation sociaux qui étaient jusque-là à l'œuvre.

2. Cet article s'appuie sur les travaux menés par l'auteur dans ces contextes : en milieu rural, ils se sont déroulés en France (Ladefroux et al., 1993) et en Espagne (Petit, 2004a) ; dans des quartiers en périphérie urbaine, en France (Petit et al., 1997) ; dans des espaces en crise, dans différents pays d'Amérique latine - Argentine, Colombie, Brésil, Mexique (Petit, 2008).

3. El libro azul. À paraître dans un recueil d'autobiographies de lecteurs, Mexico.

4. Des enseignants ou bibliothécaires travaillant dans des espaces où l'accès au livre n'est pas donné ont été incités à questionner leur propre rapport aux livres, à penser leur parcours et à écrire leurs souvenirs. Certains ont également recueilli des récits auprès des publics avec qui ils travaillent. D'autres récits ont été recueillis directement par l'auteur.

5. L'importance de la grand-mère dans les souvenirs de lecture évoqués en Amérique latine est saisissante.

6. Il y a quelques années, C. Baudelot, M. Cartier et C. Détrez (1999) avaient suivi pendant quatre ans une cohorte de 1200 adolescents. La première année, la part de ceux ayant déclaré avoir lu la veille avant de s'endormir atteignait $70 \%$ pour ceux issus de catégories favorisées, $60 \%$ pour les catégories moyennes, $50 \%$ pour les milieux défavorisés (Détrez, 2000, p. 183). Certes, ces taux diminuaient sensiblement au fil des années suivantes, avec le passage au lycée. Mais il reste étonnant qu'une majorité de collégiens choisissent la compagnie d'un livre avant de s'endormir, alors que le visuel est si présent dans leurs vies.

7. On pense là à toute la théorisation psychanalytique sur le Moi-peau et les enveloppes psychiques. Voir Anzieu, 1995.

8. R. Bradbury, Farhenheit 451, Paris, Gallimard Folio, 2000.

9. Ce n'est pas une simple métaphore. À la fin de sa vie, Freud avait noté, de façon elliptique, « la psyché est étendue, elle n'en sait rien » (Résultats, idées, problèmes II, Paris, PUF, 1985, p. 288). Il n'avait cessé de revenir à la spatialité, par exemple en concevant l'appareil psychique comme une topique. D. W. Winnicott reprend cette idée en lui donnant une place plus centrale.

10. C'est dire à quel point, dans cette perspective, les ressources culturelles sont vitales. Pour parler comme D. W. Winnicott, chacun tente de jouer avec ce qu'il trouve, des morceaux de bois ou des quatuors de Beethoven. Ce qui ne veut pas dire que tout soit équivalent ou substituable, loin de là.

11. Voir notamment les ouvrages de D. W. Winnicott, W. R. Bion, D. Meltzer, D. Stern, R. Diatkine, M. Bonnafé.

12. Tentatives qui peuvent aussi prendre la forme de la domestication et du contrôle de la lecture... 


\section{RÉSUMÉS}

L'étude des pratiques de lecture dans des espaces en crise (qu'ils soient le cadre de guerres ou de violences répétées, de déplacements forcés de populations ou de déqualifications économiques rapides), ou dans des quartiers stigmatisés en périphérie urbaine, conduit à questionner les acceptions courantes du quotidien et de ses territoires, et à s'étonner de leur réduction fréquente au banal ou au proche. Sans points d'échappée vers un ailleurs, un autre temps, d'autres registres de la langue, le proche serait peut-être inhabitable.

The study of reading practices in areas in crisis (whether scenes of wars or repeated violence, of forced population displacements or rapid economic disqualifications) or stigmatized neighbourhoods on urban outskirts leads us to question commonly accepted beliefs about everyday life and its territories and to wonder at their frequent reduction to something banal or immediate. Indeed, without escape routes towards another reality, another time, other language registers, the immediate reality might be uninhabitable.

\section{INDEX}

Mots-clés : quotidien, lecture, espace périurbain, pratiques culturelles, espace public, espace privé

Index géographique : France

\section{AUTEUR}

\section{MICHÈLE PETIT}

Anthropologue, Ingénieur de recherche, Ladyss (Laboratoire dynamiques sociales et recomposition des espaces), CNRS, Université de Paris 1 ,

2, rue Valette,

75005 - Paris

pettmic@univ-paris1.fr 